\title{
STRUCTURE OF OJ 287 FROM GEODETIC VLBA DATA
}

\author{
Claudio E. Tateyama ${ }^{1}$ \\ CRAAM-INPE, Universidade Presbiteriana Mackenzie, Rua da Consolação 896, 01302-000, São Paulo, SP, Brazil \\ AND \\ KerRy A. Kingham \\ US Naval Observatory, Earth Orientation Department, 3450 Massachusetts Avenue, Washington, DC 20392 \\ Received 2003 September 5; accepted 2004 February 25
}

\begin{abstract}
We have used the Radio Reference Frame Image Database (RRFID) data of the US Naval Observatory (USNO) at $8 \mathrm{GHz}$ to study the structure of OJ 287. The data comprise Very Long Baseline Array (VLBA) observations made during the epochs from 1994 July to 2002 January. The imaging of these data reveals that the position angle of the more collimated unresolved jet rotated clockwise $\sim 30^{\circ}$ in $8 \mathrm{yr}$. The best explanation for this result is given by a ballistic precessing model. The aperture angle of the precessing cone is $3^{\circ}$, the axis of the precessing cone forms an angle of $11^{\circ}$ with the line of sight and has a projected position angle of $-107^{\circ}$ in the plane of sky. The outer structure $(\sim 1.5 \mathrm{mas})$ of the source is also consistent with a ballistic precessing jet if the velocity of the ballistic component decreases with distance.
\end{abstract}

Subject headings: BL Lacertae objects: individual (OJ 287) — galaxies: jets — radio continuum: galaxies

\section{INTRODUCTION}

OJ 287 is a well-studied BL Lac object and distinguished in the optical by its series of prominent flares, which recur with a period of $11.6 \mathrm{yr}$ (Sillanpää et al. 1988). OJ 287 is also a remarkable radio source. High-frequency VLBI observations reveal a core-jet structure with a size not much greater than 1.5 mas in the westerly direction and evidence of bending to the southwest. At a slightly larger scale of about a few tens of mas as seen on maps at low frequencies, particularly at $2 \mathrm{GHz}$ (Fey et al. 1996; Fey \& Charlot 1997, 2000), this southwest structure dominates the source. At large scale as shown by Very Large Array (VLA) observations (Kollgaard et al. 1992; Perlman \& Stocke 1994), OJ 287 reveals an extended structure of $15^{\prime \prime}$ that appears near the position angle (P.A.) of the parsec jet; however, the connection of the parsec with the kiloparsec structure is not clear.

On the parsec scale, OJ 287 is a well-known superluminal source. VLBI observations made in 1981 by Roberts et al. (1987) show a superluminal component moving at a P.A. of $-116^{\circ}$. Since then, the superluminal components have shown a P.A. ranging from $-80^{\circ}$ to $-120^{\circ}$. Vicente et al. (1996) interpreted this result in terms of helical structure. A later study from geodetic VLBI data made by Tateyama et al. (1999), covering a period of almost 10 years, also showed a large variation of P.A. but did not find a clear case for helical structure; instead, a particular set of data observed during 1996 showed components of the jet moving almost ballistically at a P.A. of about $-90^{\circ}$. This result has been confirmed by Homan et al. (2001) in VLBA observations at 15 and $22 \mathrm{GHz}$ obtained in the same year. However, in addition to the moving components they found that the inner and the outer components had slower proper motions. Similar results were obtained by Jorstad et al. (2001) in VLBI observations at $43 \mathrm{GHz}$, who reported stationary components near the core and at 1.2 mas, which could be the result of bending in helical structure. An alternative explanation for the variation of P.A. was given by Abraham (2000), who suggested a ballistic precessing jet.

\footnotetext{
${ }^{1}$ INPE, São José dos Campos, SP, Brazil.
}

This work provides further analysis on the structure of OJ 287 by imaging the geodetic VLBA data at $8 \mathrm{GHz}$. The main result of this work is based on the discovery of the clockwise P.A. rotation of the jet with time. This result and the change in morphology of the source with time are discussed in terms of a precessing jet with the jet itself having ballistic or helical motion.

\section{OBSERVATIONS AND DATA ANALYSIS}

We have used data from the Radio Reference Frame Image Database (RRFID) of the US Naval Observatory (USNO) at $8 \mathrm{GHz}$. Observations were made using an array consisting of geodetic antennas along with the 10 antennas of the Very Long Baseline Array (VLBA; Napier et al. 1994) of the National Radio Astronomy Observatory ${ }^{2}$ (NRAO). The observations were processed with the VLBA correlator at the Array Operations Center in Socorro, New Mexico. All observations were obtained with dual-frequency VLBA receivers (Rogers et al. 1983 ) at both $\mathrm{X}$ and $\mathrm{S}$ band (centered at 8.6 and $2.3 \mathrm{GHz}$, respectively). The $\mathrm{X}$ band back end consists of four individual channels of $8 \mathrm{MHz}$ bandwidth spanning the range 8.41$8.90 \mathrm{GHz}$. The sources were observed using short duration ( $\sim 3$ minutes) "snapshots" over a number of different hour angles to maximize the $(u, v)$-plane coverage. Table 1 lists all observations used in this work: column (1) gives the epochs of the observations, column (2) the antennas participating in the experiments, column (3) the peak brightness of the images, column (4) the interferometric dirty beams, and column (5) the P.A.s of the beams measured from north through east. The map of 1996 is not included in the work because of poor visibility data, and the maps of 1997 January 10 and 11 are well represented by the map of January 31, which has a higher data quality. The dynamic range as given by the ratio of the peak flux per beam to the lowest positive contour in the images is $\sim 1200: 1$.

\footnotetext{
2 The National Radio Observatory is a facility of the National Science Foundation operated under cooperative agreement by Associated Universities, Inc.
} 
TABLE 1

VLBA 8 GHz OBSERVATIONS

\begin{tabular}{|c|c|c|c|c|}
\hline $\begin{array}{l}\text { Epoch } \\
\text { (1) }\end{array}$ & $\begin{array}{l}\text { Antennas } \\
\text { (2) }\end{array}$ & $\begin{array}{l}\text { Peak Brightness } \\
\left(\mathrm{Jy} \mathrm{beam}^{-1}\right) \\
\text { (3) }\end{array}$ & $\begin{array}{l}\text { Synthesized Beam } \\
(\text { mas } \times \text { mas }) \\
(4)\end{array}$ & $\begin{array}{c}\text { Beam Angle } \\
(\text { deg }) \\
(5)\end{array}$ \\
\hline 1994 Jul 08 .. & VLBA & 1.42 & $1.84 \times 1.23$ & 7.4 \\
\hline 1995 Apr $12 \ldots \ldots \ldots \ldots$ & VLBA & 0.89 & $1.86 \times 0.99$ & -7.2 \\
\hline 1995 Oct 13 & VLBA & 1.56 & $1.74 \times 1.04$ & -10.2 \\
\hline 1996 Apr $24 \ldots \ldots \ldots$ & VLBA & 1.19 & $2.0 \times 0.82$ & -12.1 \\
\hline 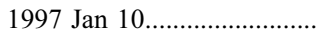 & VLBA & 0.71 & $1.69 \times 0.95$ & 0.7 \\
\hline 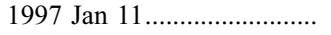 & VLBA & 0.72 & $1.78 \times 1.03$ & -1.8 \\
\hline 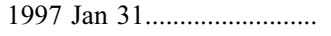 & VLBA + 6GA & 0.78 & $1.38 \times 0.75$ & -8.0 \\
\hline 1997 Mar 31 .......................... & VLBA + 6GA & 0.77 & $1.29 \times 0.70$ & -6.2 \\
\hline 1997 May 19......................... & VLBA + 6GA & 0.82 & $1.45 \times 0.70$ & -7.9 \\
\hline 1997 Jul 24 & $\mathrm{VLBA}+6 \mathrm{GA}$ & 1.03 & $1.43 \times 0.75$ & -5.6 \\
\hline 1997 Dec 17 & $\mathrm{VLBA}+7 \mathrm{GA}$ & 1.16 & $1.35 \times 0.73$ & -1.1 \\
\hline 1998 Feb 09 & $\mathrm{VLBA}+7 \mathrm{GA}$ & 1.24 & $1.26 \times 0.72$ & -8.2 \\
\hline 1998 Apr $15 \ldots \ldots \ldots$ & $\mathrm{VLBA}+7 \mathrm{GA}$ & 1.68 & $1.44 \times 0.67$ & -9.2 \\
\hline 1998 Jun 24 & $\mathrm{VLBA}+7 \mathrm{GA}$ & 1.44 & $1.36 \times 0.66$ & -8.7 \\
\hline 1998 Aug $10 \ldots \ldots$ & $\mathrm{VLBA}+7 \mathrm{GA}$ & 1.31 & $1.31 \times 0.69$ & -8.7 \\
\hline 1998 Dec 21 & VLBA + 6GA* & 1.08 & $1.14 \times 0.77$ & -8.4 \\
\hline 2002 Jan $16 \ldots \ldots \ldots \ldots \ldots \ldots \ldots$ & VLBA + 8GA & 1.24 & $1.15 \times 0.58$ & -8.4 \\
\hline
\end{tabular}

Notes.-VLBA consists of 10 antennas of $25 \mathrm{~m}$, and GA stands for Geodetic Antennas; 6GA = Gc, Gn, Kk, Mc, On and Wf; 7GA $=6 \mathrm{GA}+\mathrm{Ny} ; 6 \mathrm{GA}^{*}=7 \mathrm{GA}-\mathrm{On} ; 8 \mathrm{GA}=7 \mathrm{GA}+\mathrm{Ts}+\mathrm{Wz}-\mathrm{Gn}$. Gc is Gilcreek (Alaska, $26 \mathrm{~m}$ ), Gn is NRAO20 (Greenbank, $20 \mathrm{~m}$ ), Kk is Kokee (Hawaii, $20 \mathrm{~m}$ ), Mc is Medicina (Italy, $32 \mathrm{~m}$ ), Ny is Ny Alesund (Norway, $20 \mathrm{~m}$ ), On is Onsala (Sweden, $20 \mathrm{~m}$ ), Ts is Tsukuba (Japan, $32 \mathrm{~m}$ ), Wf is Westford (Massachusetts, $18 \mathrm{~m}$ ), and $\mathrm{Wz}$ is Wettzell (Germany, $20 \mathrm{~m}$ ).

The data were calibrated and fringed using standard routines from the AIPS software package, and the images were obtained using DIFMAP (Shepherd 1997). Initially, a window of 2 mas in the horizontal axis (east-west) and 1 mas in the vertical axis (north-south) was placed on the brightest point of the source, centered at $\sim 0.5$ mas from the east side of the window to avoid the appearance of a spurious counter jet. On this initial stage of imaging, the main features of the source, such as the more collimated jet structure that shows the gradual change of P.A. over time and the curving structure of $\sim 1.5$ mas in length for maps observed in 1998, were well defined in the maps.

Outside this area, especially on the southwest region of the source, the structure is dominated by a weak extended emission, showing a fuzzy morphology where the maxima could be better explained by the dispersion of the intrinsic system noise than by a real change in the structure. Even after careful windowing, there remain features that are inconsistent between images in the sequence. These do not affect the main structure of the source as seen in the maps. The data were initially phase self-calibrated and imaged using uniform weighting before switching to natural weighting. The map was restored to a resolution of $0.5 \times 0.5$ mas, which is comparable to about the smallest beam minor axis of these VLBA observations. In fact, the more detailed structure corresponds with that in published data using Gaussian fitting.

\section{RESULTS AND DISCUSSION}

Figure 1 shows the VLBA maps at $8 \mathrm{GHz}$ from 1994 April to 2002 January. The main feature of the maps is emphasized by the thick contour line on the maps, which rotates in a clockwise direction on the sequence of maps. The jet appears initially at $\sim$ P.A. $-90^{\circ}$ from 1994 July to 1997 January. The direction of the jet shifts in a clockwise direction from 1997 January through 1998 December until reaching the P.A. of $-120^{\circ}$ in the 2002 January map. Meanwhile, the morphology seen in the images changes. The morphology of the early maps (1994 July1995 October) is distinguished by a symmetrical north-south lobe at $\sim 1.5$ mas from the core. In fact, this might simply be the result of a more symmetrical form compared with the more asymmetric northern and southern structures of the other maps in Figure 1. The symmetrical structure may be unusual for a jet showing a large radial expansion, but the features seem real and not artifacts of the imaging process. All maps (except 2002 January) show a small protuberance to the northwest of the source. At the same time they show an extended southwest structure, notably on the maps from 1998 April to December. In the 1997 May and December maps, however, this southwest structure does not show up so clearly. The 2002 January map shows a single elongated structure in the southwest direction, with a secondary component a few mas apart in the same direction, exhibiting a distinct morphology compared to other epochs, which appear to be more like a wide cone structure. In addition to this, the orientation of the jet appears to be a little more rotated in the southerly direction.

Figure 2 shows a comparison of the structure between our restored 0.5 mas map and VSOP (VLBI Space Observatory Program) component positions (Gabuzda \& Gómez 2001). The VLBA data (1998 December) were obtained about two months earlier than the VSOP data. The positions of the VSOP components agree well with VLBA components, indicating that the finer structures, as shown by our restored 0.5 mas VLBA map, are not artifacts of the imaging.

\section{HELICAL JET}

In order to examine the features present on the maps, we assumed that the jet as a whole is precessing, with components of the jet moving in a helical structure. In modeling the source structure, we have considered the hydrodynamic helical jet model described by Hardee (1987). This model has been applied to the BL Lac objects OJ 287 (Vicente et al. 1996), 

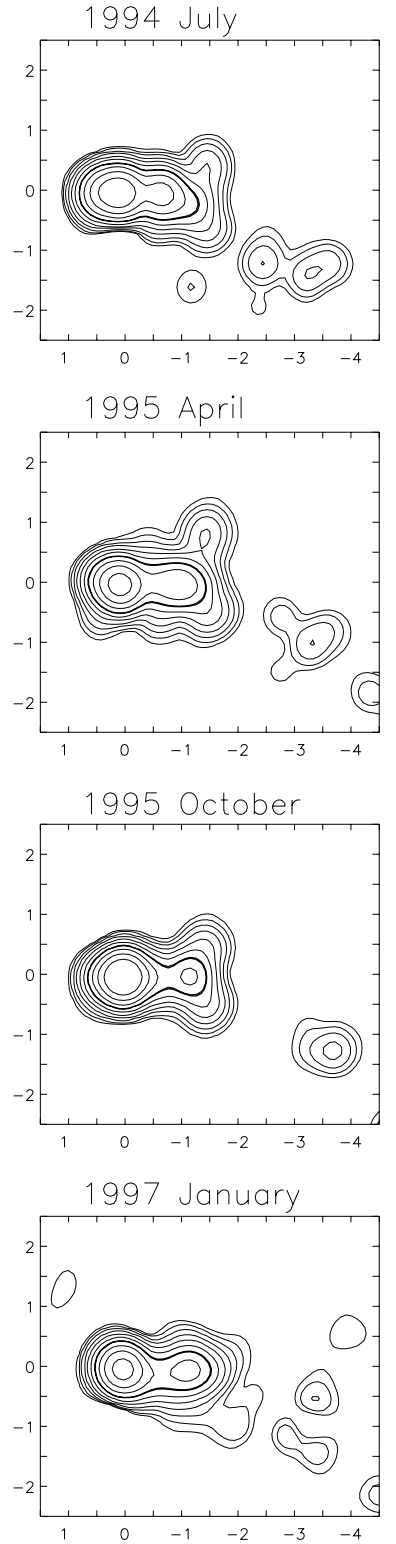

1997 March

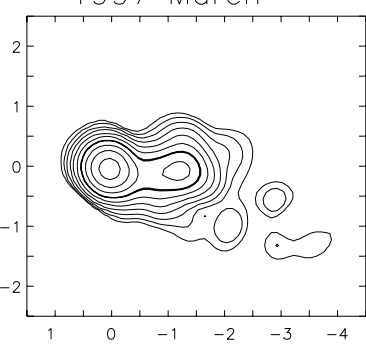

1997 May
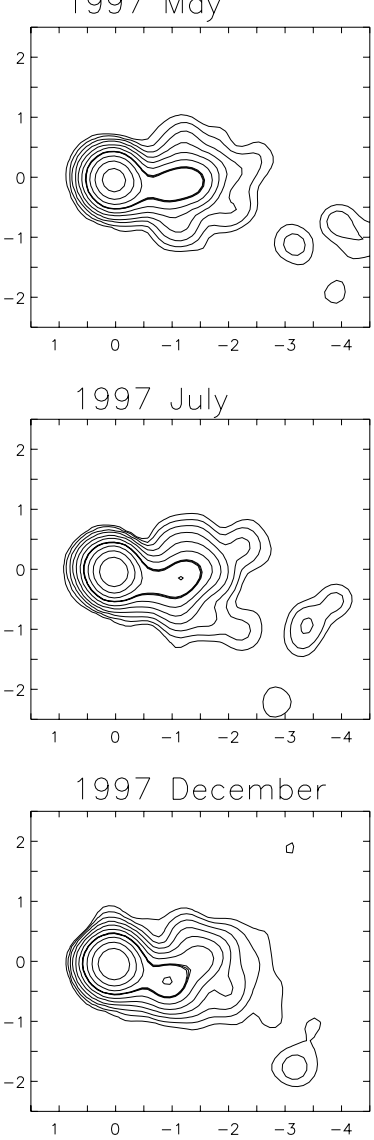
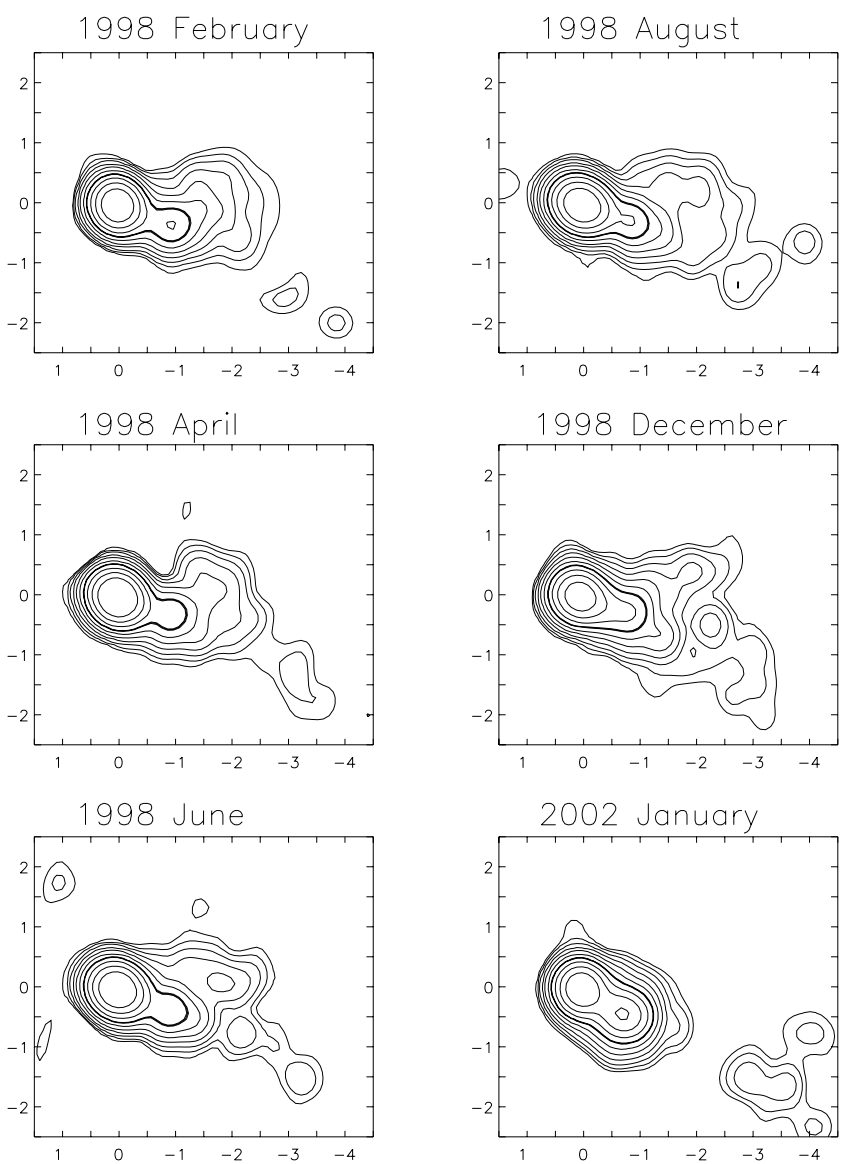

FIG. 1.-VLBA $8 \mathrm{GHz}$ images of OJ 287. Contour levels are $(1,2,4,8,16,32,64,128,256$, and 512$) \times 0.001$ Jy beam ${ }^{-1}$. The thick line on the contour levels corresponds to the level 64 , in order to emphasize the rotation of the jet orientation. The restored circular beam is 0.5 mas.

BL Lac (Tateyama et al. 1998; Denn et al. 2000), and 1803+784 (Tateyama et al. 2002). For this work, the best helical fitting was obtained using the adiabatic jet model.

The helical feature describes a spiral on the $(x, y)$-plane with amplitude

$$
A=A_{1} \exp \left\{2 d_{1}\left[1-\left(\frac{z_{1}}{z}\right)^{1 / 2}\right]\right\}
$$

and phase

$$
\phi=\phi_{1} \pm 2\left[1-\left(\frac{R}{R_{1}}\right)^{1 / 2}\right] \frac{\tan \Delta}{\alpha},
$$

with

$$
\tan \Delta=\frac{2 \pi A_{1}}{\lambda_{1}},
$$

where $R=\Psi\left(z+z_{0}\right)$, with the axis of the helical jet on the $z$-coordinate axis making an angle $\chi$ with the observer's line of sight. $\Psi$ is the half-opening angle of the jet and $z_{0}$ is the origin of the cone; $A_{1}, \phi_{1}, \lambda_{1}$ and $R_{1}$ are the amplitude and phase of the helix, the helical wavelength, and the radius of the jet, respectively, at distance $z_{1}$ from the origin; $d_{1}$ expresses the rate at which the helical amplitude grows, also evaluated at $z_{1}$. Assuming that $A / R=\xi$ at $z_{1}, \alpha=\xi \Psi=\xi R_{1} /\left(z_{1}+z_{0}\right)$ and $\Delta=\arctan \left(2 \pi A_{1} / \lambda_{1}\right)$, which are taken as the opening angle and pitch angle of the helical jet. Figure 3 illustrates the main parameters of the adiabatic model. This model shows a rapid increase of the helical amplitude near the core, but soon the amplitude becomes almost constant. Unlike isothermal jets, where the helical jet can be described moving on the surface of a cone of constant opening angle and pitch angle, in the adiabatic case we take these values at $z_{1}$.

The helical model was obtained by fitting the morphology of each map, varying only the viewing angle of the helical jet, followed by a rotation of the helical axis (P.A.) in a clockwise 


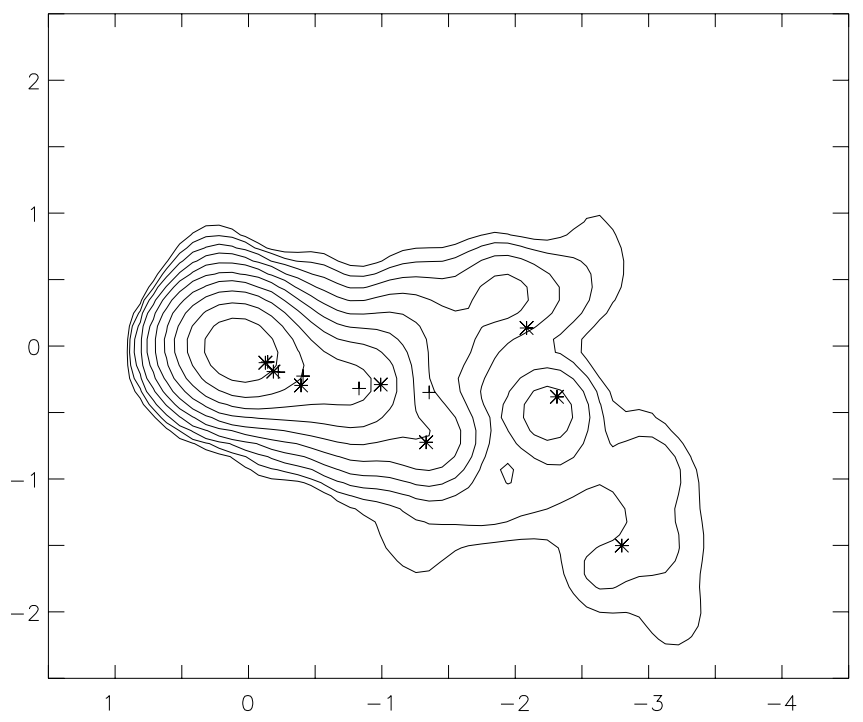

FIG. 2.-Comparison of VSOP components on the 1998 December VLBA map. VSOP data points are represented by asterisks and VLBI data at $22 \mathrm{GHz}$ by crosses.

direction. The fixed helical parameters are given by $\alpha=1.10$ and $\Delta=12^{\circ} .0$. The jet parameters are given by $\Psi=0.69$, $R_{1}=0.51 \mathrm{mas}, \lambda_{1}=24.1 \mathrm{mas}, A_{1}=0.82 \mathrm{mas}, \phi_{1}=-0.9$, $z_{0}=0.5$ mas, $z_{1}=42.0$ mas, $d_{1}=1.2$, and $\xi=1.6$. The viewing angle $\chi$ and the P.A. of the jet for each epoch are listed on Table 2.

Figure 4 shows the helical curves superposed on the VLBA maps of Figure 1. Here the symmetrical north-south morphology pattern in the 1994 July-1995 October maps, as well as the structure in the form of arc on the maps of 1998 February1998 December, are well described by a helical ridgeline. The good agreement of this arched structure, along with the southwest extension, gives strong support in favor of a helical structure. In this way, the outer weak extended western side of the source can be considered as a structure formed from distinct parts of the helical jet, a combination of emission coming from an outer loop that is bending toward our line of sight when passing near the inner loop. The other side of this outer loop

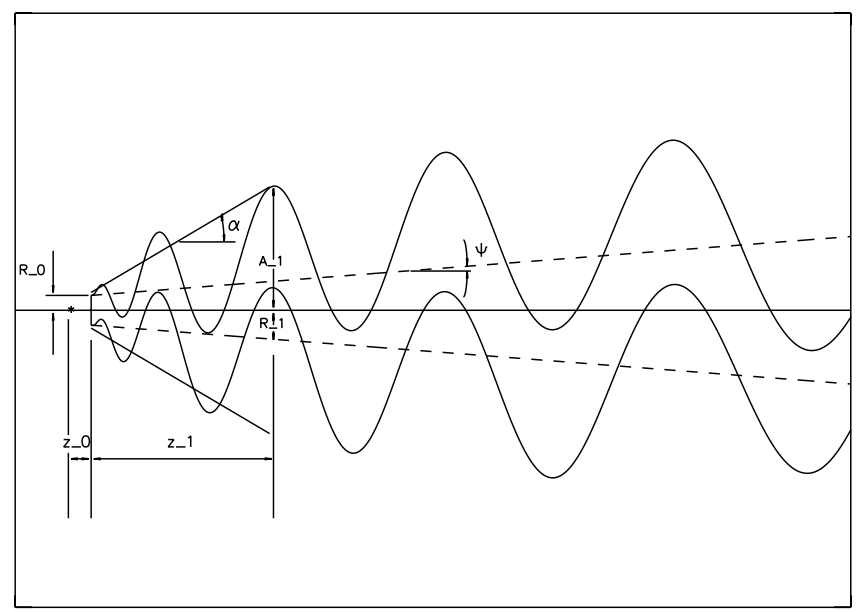

FIG. 3.- Helical and jet parameters of the adiabatic jet model. Dashed lines represent the shape of the jet cone of constant opening angle $\alpha$ and solid lines represent the shape of the helical amplitude.
TABLE 2

Helical Parameters

\begin{tabular}{|c|c|c|c|}
\hline Epoch & $\begin{array}{l}\text { Viewing Angle } \\
\text { (deg) }\end{array}$ & $\begin{array}{l}\Delta \text { P.A. }^{1} \\
(\text { deg) }\end{array}$ & $\begin{array}{l}\text { P.A. } \\
\text { (deg) }\end{array}$ \\
\hline 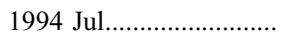 & 3.7 & 0.0 & -96.5 \\
\hline 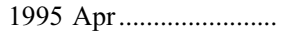 & 3.7 & 5.0 & -91.5 \\
\hline 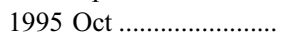 & 3.7 & 7.0 & -89.5 \\
\hline 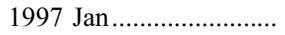 & 3.7 & 6.5 & -90.0 \\
\hline 1997 Mar........................... & 3.8 & 5.5 & -91.0 \\
\hline 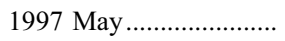 & 4.2 & 3.0 & -93.5 \\
\hline 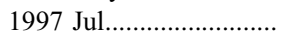 & 4.8 & 0.0 & -96.5 \\
\hline 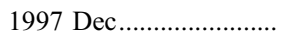 & 5.0 & -3.0 & -99.5 \\
\hline 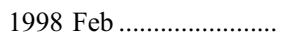 & 5.5 & -3.5 & -99.5 \\
\hline 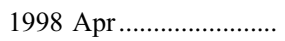 & 5.5 & -4.0 & -100.5 \\
\hline 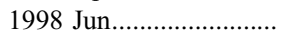 & 5.5 & -4.5 & -101.0 \\
\hline 1998 Aug ....................... & 5.5 & -4.5 & -101.0 \\
\hline 1998 Dec & 6.0 & -5.0 & -101.5 \\
\hline 2002 Jan & 14.0 & -25.0 & -121.5 \\
\hline
\end{tabular}

${ }^{1}$ Variation of position angle of the component.

(western side), where no jet emission is seen, is also consistent with components moving away from our light of sight. Finally, the largest change in morphology in the 2002 January map shows more of a straight rectilinear structure, suggestive of a large viewing angle $\chi$.

Stationary components can be taken as a sign of helical structure. Homan et al. (2001) made 15 and 22 GHz VLBA observations of OJ 287 that show that the inner and outer components have lower proper motions. Jorstad et al. (2001), in VLBA observations at $43 \mathrm{GHz}$, have also reported stationary components near the core and at 1.2 mas, which appear to indicate bending in the helical structure. In fact, the 43 and $8 \mathrm{GHz}$ observations may reveal different aspects of the source. While higher frequencies such as $43 \mathrm{GHz}$ emphasize the compact discrete components, indicating the position of the shock-induced dense regions, i.e., the bends on the jet, at lower frequencies observations may trace a continuous pattern, with material streaming along the jet as indicated by the $8 \mathrm{GHz}$ VLBA maps. This effect may not simply be due to the lower resolution of $8 \mathrm{GHz}$ data in relation to $43 \mathrm{GHz}$, since VSOP data $(5 \mathrm{GHz})$ are also dominated by extended features (see Fig. 3 of Gabuzda \& Gómez 2001).

\subsection{Precession Model}

So far, the viewing angle $\chi$ and P.A. of each map have been obtained by fitting a helical structure to the morphology of the maps. The variation of viewing angle and P.A. suggests that the components of the jet are moving in a helical jet but superimposed on the precession of the jet itself. Here we want to obtain this precessing component.

We consider a relativistic jet precessing with a constant velocity $\omega$ and a period $T$ around a axis that forms an angle $\chi_{0}$ with the line of sight and having a projected angle $\eta_{0}$ in the plane of the sky. For an aperture angle of the precessing cone $\Omega$, the instantaneous position of the jet with time $t$ is represented by the angles $\chi$ and $\eta$ (P.A.) as given by Abraham (2000),

$$
\begin{gathered}
\tan \eta(t)=\frac{Y}{X}, \\
\sin ^{2} \chi(t)=X^{2}+Y^{2},
\end{gathered}
$$



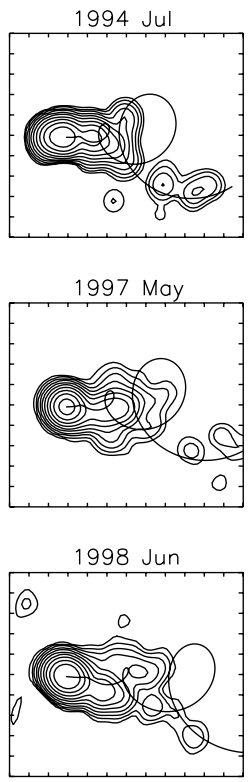

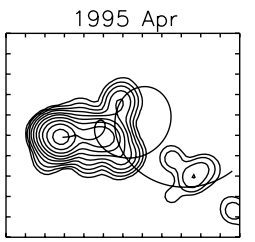

1997 Ju

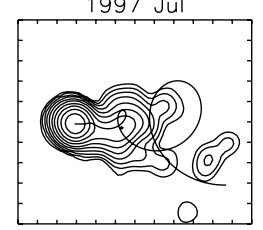

1998 Aug

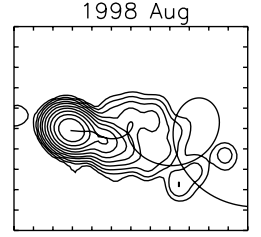

1995 Oct

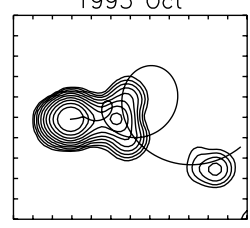

$1997 \mathrm{Dec}$

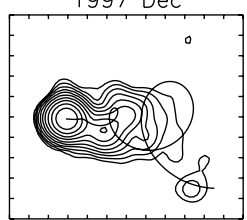

$1998 \mathrm{Dec}$

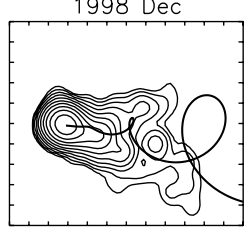

1997 Jan

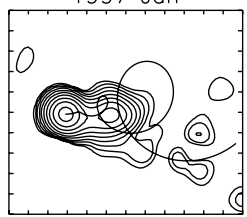

1998 Feb

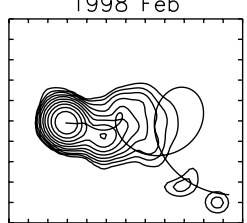

2002 Jan

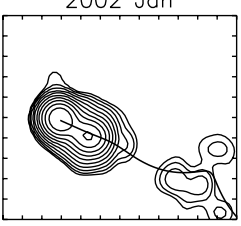

FIG. 4.- Helical curves superposed on the VLBA maps of Fig. 1. The solid line represents the best helical model to describe the radio features.

with

$$
\begin{aligned}
X= & \left(\cos \Omega \sin \chi_{0}+\sin \Omega \cos \chi_{0} \sin \omega t\right) \cos \eta_{0} \\
& -\sin \Omega \cos \omega t \sin \eta_{0}, \\
Y= & \left(\cos \Omega \sin \chi_{0}+\sin \Omega \cos \chi_{0} \sin \omega t\right) \sin \eta_{0} \\
& +\sin \Omega \cos \omega t \cos \eta_{0} .
\end{aligned}
$$

Figure 5 shows 2 precessing models for the helical parameters. The curves represent the precession of the jet with no helical motion. For a given period $T$, the shape of the curve of P.A. with time is given by the ratio of the aperture angle of the cone, $\Omega$, to the angle that the jet axis makes with the line of sight, $\chi_{0}$. Both models were made to have the same curve for P.A. with time. The best model is obtained with an aperture angle of 1.5 , which shows a good fit for all data $(\chi)$ except the data corresponding to 2002 January. However, to fit this discordant data we would need a larger aperture angle of 4.8 , which displaces the curve up as shown in the figure. Thus, a precessing jet could account for the P.A. and viewing angle $\chi$ obtained from helical fitting from 1994 July until 1998 December. After this period the viewing angle, instead of decreasing as would be expected for a precessing jet, continues to increase, indicating that the jet is moving away from our line of sight. In this model we have a precessing jet, with components of the jet moving in a helical pattern that works well from 1994 to 1998 but not with 2002 data. This suggests that either something unusual occurred after 1998 December that caused a departure from regular precessing motion, or the jet could still be precessing in a regular way but the components of the jet are not moving in a helical pattern. The period of precession derived from helical fitting of $13.6 \mathrm{yr}$, which is longer than the observed periodicity of $11.6 \mathrm{yr}$, could be a further argument against the helical structure. Another explanation for our VLBA images is a precessing nozzle where the jet components appear as ballistic knots instead of helically moving components.

\section{BALLISTIC MODEL}

Until this point, we have examined the results of the observations in terms of a helical precessing jet. We have as- sumed that the jet was precessing and that at the same time the components of the jet themselves were moving in a helical pattern. However, we have found that the viewing angle of the helical jet, as derived from the morphology of the map of 2002 January, is not consistent with the viewing angle predicted by the helical precessing model.

In this section, we examine the data in terms of ballistic components of a precessing nozzle. Returning to Figure 1, the northwest lobe that appears in 1998 August-December can also be reinterpreted as a combination of older and more distant jet components, with more recent components being gradually ejected at higher P.A.s in order to produce the curved structure shown on the maps. As the precession continues, the structure of the jet may straighten up and eventually invert its pattern from convex to concave curvature. The 2002 January map may be in this period. Other stages of the precession may be

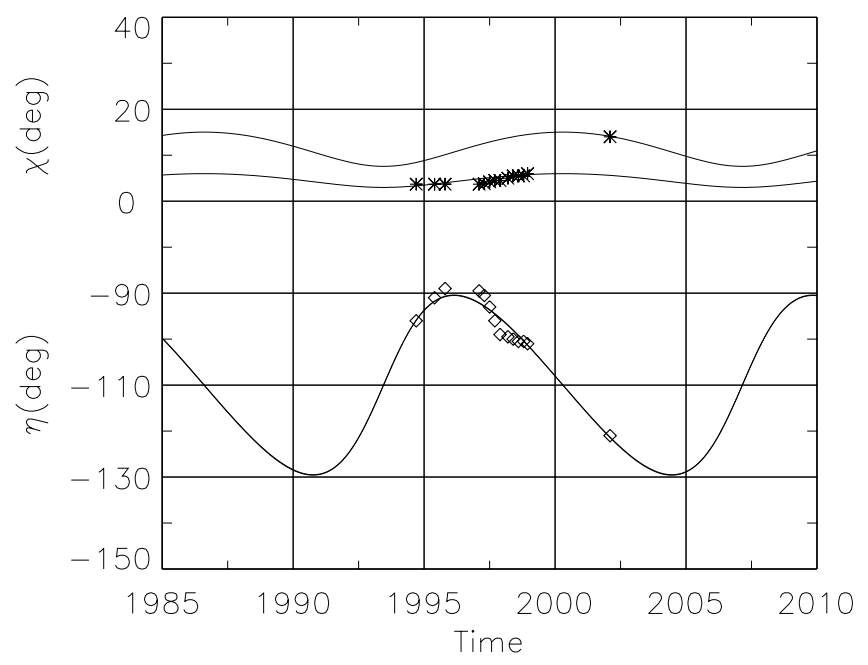

FIG. 5.-Precession model for a helical jet. The curve on the upper side of the figure shows the viewing angle with time and the curve on the lower side of the figure shows the P.A. of the jet with time. The diamonds and asterisks indicate the P.A. and viewing angle, respectively, derived from helical fits. This curve was obtained for $T=13.7 \mathrm{yr}, \Omega=1.5$ and $3.8^{\circ}, \chi=4.5$ and $11^{\circ} .4$, and position angle $\eta=-110^{\circ}$. 


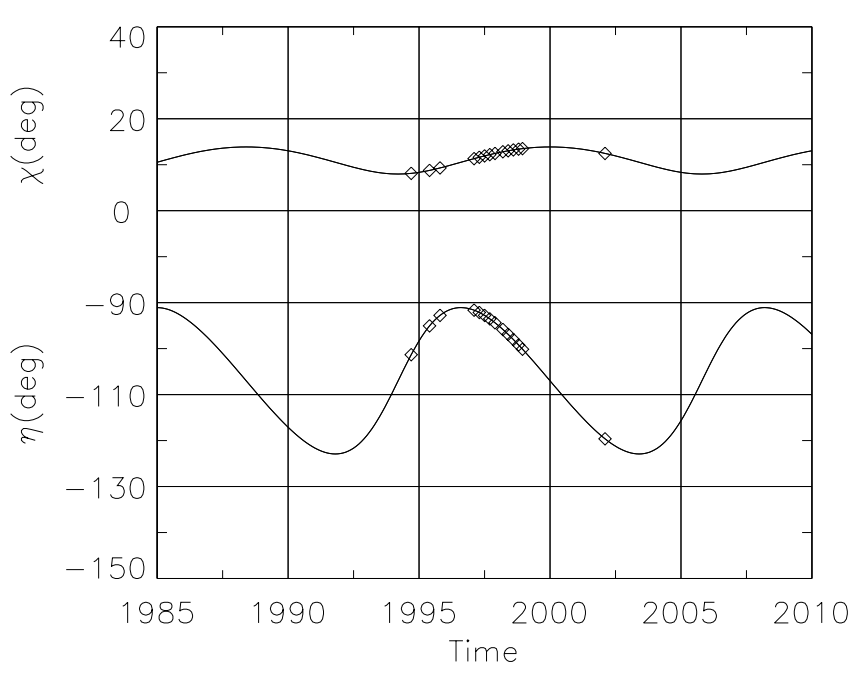

FIG. 6.-Precession model for a ballistic jet. The precessing parameters are $\Omega=3^{\circ}, \chi_{0}=11^{\circ}, \eta_{0}=-107^{\circ}$, and $T=11.6 \mathrm{yr}$. The diamonds indicate the points on the precessing curve corresponding to the epochs of the VLBA observations.

depicted on the maps of 1994 July and 1995 April; if we ignore the northwest extension of the map, the structure of the source within 1.5 mas shows an inverted jet curvature compared to 1998 April-December maps.

However, if the curved structure of 1998 April-December is described in terms of a ballistic precessing nozzle the southwest component cannot be explained as a single extension of the jet, since the nozzle would have just started to open the jet in this direction. Similar difficulties appear in the north-south structure of 1994 July to 1995 October. While the structure near the core and the southern extension agree well in terms of ballistic precessing jet, the north extension of this structure does not fit this picture. The existence of these structures within the ballistic precessing jet is not clear. We may simply assume the southwest component as a transition point between the ballistic core jet to the outer jet, which could even be related to the kiloparsec structure seen on VLA maps (Kollgaard et al.
1992; Perlman \& Stocke 1994). While the northern extension of the maps, especially the 1994 July and 1995 April maps, appears to be less convincing as a true structure within the ballistic precessing jet, the southern component could be part of a larger structure that extends to the southwest of the source.

Now let us examine how the structure of the source would appear in a simple ballistic precessing model. For a ballistic jet, the radial distance $r$ with time $t$ can be written as

$$
r=\mu f_{\chi} t
$$

where

$$
f_{\chi}=\frac{\beta_{\chi}}{\beta_{0.6}}=\frac{1}{\beta_{0.6}} \frac{\beta \sin \chi}{1-\beta \cos \chi},
$$

$\beta_{0.6}$ is the apparent velocity corresponding to a proper motion

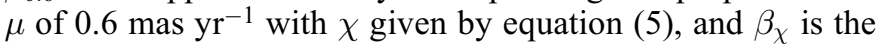
apparent velocity, which depends on the velocity of the jet $\beta$ and the viewing angle $\chi$.

The ridgeline formed by a combination of a precessing nozzle with knots having ballistic motion can be described by

$$
\begin{aligned}
& x=r \cos \eta, \\
& y=r \sin \eta,
\end{aligned}
$$

with $\eta$ given by equation (4).

Figure 6 shows the precessing model based on the curve given by equations (10) and (11) to fit the structures of the maps and shown in Figure 7. Here in the ballistic precessing jet the structure of the source is more sensitive to the rate of change of P.A. than the change of viewing angle in the helical model. However, the viewing angle still defines the morphology of the source by constraining the proper motion of the components. We have assumed a proper motion of 0.6 mas $\mathrm{yr}^{-1}$ as obtained from the VLBI observations made in 1996 (Tateyama et al. 1999). From the precessing model in Figure 6, the viewing angle at this epoch is $\sim 10^{\circ}$. Hence, for a Hubble constant of $65 \mathrm{~km} \mathrm{~s}^{-1} \mathrm{Mpc}^{-1}$ and deceleration parameter
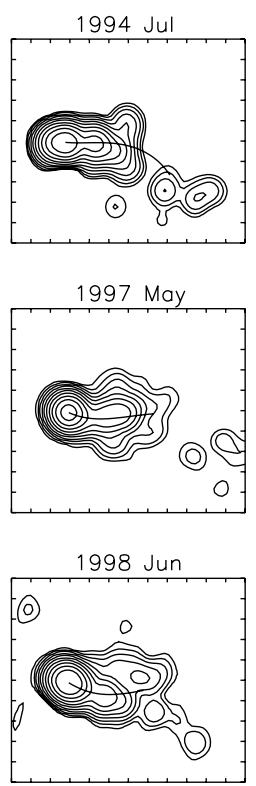

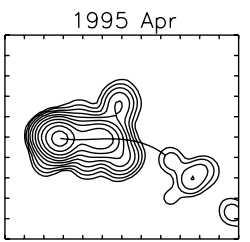

1997 Jul

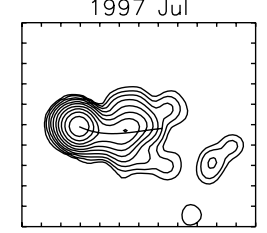

1998 Aug

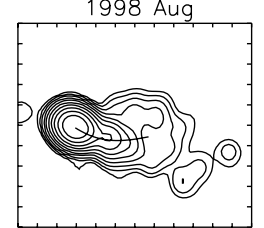

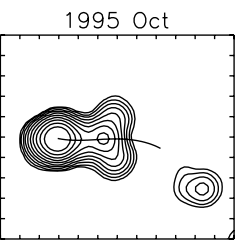

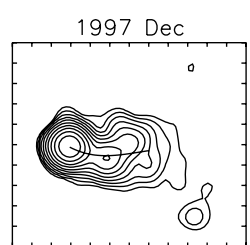

1998 Dec

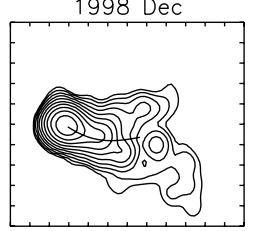

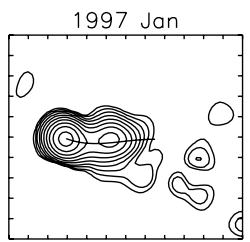
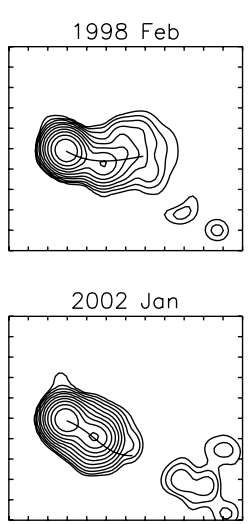
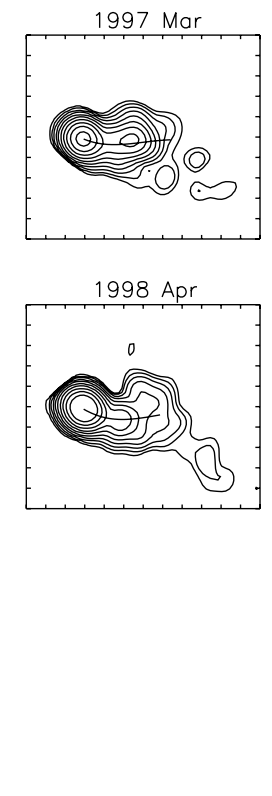

FIG. 7.-Ballistic precessing model. The line superposed on each VLBA map of Fig. 1 is obtained by a continuos ejection of knots from a nozzle that precesses as shown on Fig. 6. These knots have a ballistic motion that in combination with precession produce a ridgeline as described by eqs. (10) and (11). 
$q_{0}=0.5$, the true velocity of the jet $\beta$ is $0.998 \mathrm{c}$, which is used in equation (9).

Figure 7 shows that the ballistic precessing model describes well the structure of the jet near the core, covering almost a complete cycle of a precession. The outer structure can still be explained as a ballistic precessing jet if we assume that the ballistic components are decelerated. The shape of the ridgelines shown in the figure supports this picture. In particular, the curved structure on the 1998 April-December maps provides a good indication of deceleration. In the absence of deceleration, for the rate of change of P.A. shown on the precessing model of Figure 6 the proper motion of ballistic components should be as low as 0.3 mas $\mathrm{yr}^{-1}$ to reproduce the curved structure; however, this proper motion is too low compared with the measured proper motion of 0.6 mas $\mathrm{yr}^{-1}$ observed in 1996 (Tateyama et al. 1999) and even more inadequate if we consider the higher proper motions measured by Homan et al (2000). Also, for such low proper motion the ridgeline of the structure of the source should appear more distorted, showing a sinusoidal structure on the maps that is not observed.

\section{BLACK HOLE}

A natural explanation for the precession of the jet is to consider that the collimator of the beam is an accreting, rotating massive black hole submitted to the torque exerted by another black hole of comparable mass orbiting around it (Katz 1997), or that the accretion disk itself drives the Lense-Thirring precession of the inner disk and central compact object (Sarazin et al. 1980). Following Lu (1990), the period of precession for an accretion disk-driven jet precession model can be written as

$$
\begin{aligned}
P_{\text {orb }} \approx & 2.5 \times 10^{14} a^{1 / 2}\left(\frac{M_{*}}{M_{\odot}}\right)^{-1 / 2} \\
& \times\left(\frac{\dot{M}}{\dot{M}_{\mathrm{E}}}\right)^{-3 / 2}\left(\frac{v_{r}}{v_{\phi}}\right)^{3 / 2} \mathrm{yr},
\end{aligned}
$$

where $a$ is the black hole's dimensionless specific angular momentum $(0 \leq a \leq 1$; here we have taken $a=1), M_{*}$ is the mass in units of $10^{8} M_{\odot}, \dot{M}$ is the mass accretion rate, $v_{r}$ and $v_{\phi}$ are, respectively, the radial inflow and rotational velocity in the disk, and $\dot{M}_{\mathrm{E}}$ the Eddington-limit accretion rate for a massto-energy conversion of $10 \%$. For an extreme Kerr hole with mass $M_{*} \sim 4 \times 10^{8} M_{\odot}$, precession period $P=12 \mathrm{yr}$, and assuming a $\dot{M} / \dot{M}_{\mathrm{E}} \sim 24$, which lies on the higher rate of the quasars studied by Collin et al. (2002), it yields an inflow rate $v_{r} / v_{\phi}$ at $r_{p}$ of $\sim 510^{-8}$. The critical radius $r_{p}$ is given by

$$
\begin{aligned}
r_{p} & \approx 2.5 \times 10^{19} a^{1 / 2}\left(\frac{M_{*}}{M_{\odot}}\right)^{1 / 2}\left(\frac{\dot{M}}{\dot{M}_{\mathrm{E}}}\right)^{-1 / 2}\left(\frac{v_{r}}{v_{\phi}}\right)^{1 / 2} \mathrm{~cm} \\
& \approx 2.3 \times 10^{15} \mathrm{~cm} .
\end{aligned}
$$

In the case of a precessing disk model (Katz 1997), the orbital period of the binary can be related to the precession period of the disk by the relation

$$
P_{\mathrm{orb}}=\left[1.295 \frac{m}{M_{*}^{1 / 2}\left(M_{*}+m\right)} P_{\mathrm{prec}} a_{d}^{3 / 2} \cos \theta\right]^{1 / 2} \mathrm{yr},
$$

where $M_{*}$ and $m$ are the mass of the primary and the companion black hole, respectively, in units of $10^{8} M_{\odot} ; a_{d}$ is the radius of the accretion disk in $10^{16} \mathrm{~cm} ; \theta$ is the inclination of the disk to the orbital plane; and $P_{\text {orb }}$ and $P_{\text {prec }}$ are the orbital and precessional periods in years.

We take the observed period of $12 \mathrm{yr}$ as the precessional period. Assuming that the secondary maximum observed $1.2 \mathrm{yr}$ afterward is evidence for a nodding motion of the precessing disk, Katz (1997) derived $P_{\text {prec }} / P_{\text {orb }} \sim 5$. Now, for a mass of the primary black hole of $4 \times 10^{8} M_{\odot}$ (Liu \& Wu 2002), $m / M_{*} \sim 1$, and $\theta=3^{\circ}$ from our precessing model, equation (14) yields a size of the accretion disk $a_{d}=1.27 \times 10^{16} \mathrm{~cm}$. While a $m / M_{*} \sim 1$ gives an exceptionally massive secondary black hole, the parameters are not unreasonable for a source such as OJ 287. The difference between the original precessing disk model of Katz (1997) and the present work is that in his work the whole jet moves as it precess, while in our case the model only provides a means to change the direction of the ballistic knots. The combination of a sequence of isolated knots ejected from this precessing nozzle would exhibit the structure and the evolution of the structure seen on the VLBA maps.

One of the difficulties imposed on the precessing model is that the emission of the first of the double flares is believed to be thermal (Valtaoja et al. 2000). A natural explanation for thermal emission comes from a collision or penetration of the companion black hole by the accretion disk of the primary black hole. At the same time, such an interaction would increase the accretion rate and later produce the nonthermal flare from the jet. Thus, the double flares would be composed of a thermal emission followed by a nonthermal emission. On the other side, in a precessing jet the appearance of the flares would be produced by the Doppler boosting when the jet points in our direction. The nodding motion would account for the second flare exhibiting the double-peaked structure with both flares being synchrotron, the thermal emission being totally absent in this model. However, since the change of the P.A. of the more collimated jet seen in the VLBA maps seems to lead to precession of the jet, the flares must consequently always be synchrotron. Also, the absence of a radio counterpart, which is taken as evidence of thermal emission, should in fact be the result of synchrotron self-absorption process. A glance at the light curves of 5, 8, and $15 \mathrm{GHz}$ of the Michigan data (see, e.g., Fig. 3 of Tateyama et al. 1999) shows that the self-absorbing structure dominates the source. Besides, the radio counterpart may be weak but not absent, suggesting synchrotron emission since thermal emission from the accretion disk would not be detectable in radio. This is shown clearly in the 1994-1996 flares, particularly in the outburst of November 1994 (see Fig. 9 of Pursimo et al. 2000). While in the 1971-1972 flares there are only sparse $8 \mathrm{GHz}$ monitoring data from Michigan, for the 1983-1984 flares the outbursts profile shows a broad doublepeaked radio counterpart that becomes more predominant at higher frequencies, giving further indication of a self-absorbed source.

\section{CONCLUSION}

This work makes use of RRFID data of USNO at $8 \mathrm{GHz}$ corresponding to the observations made from 1994 July-2002 January. The main result of this work is the discovery of the rotation of the position angle of the inner, more collimated structure of the source in a clockwise direction in the sequence of maps. The source was first discussed in terms of helically moving components in a precessing jet. The change of helical structure of the maps was shown to be consistent with variation of the viewing angle, as predicted by a helical precessing 
jet from 1994 July to 1998 December; however, it fails for the structure of the 2002 January map. Even if we discard the 2002 January data, perhaps the strongest objection to helical jet is that the jet precess as a whole.

Our conclusion is that the ballistic precessing jet provides the best explanation for the structure present on the maps, especially near the core where the gradual shift of position angle of the jet structure could be the result of knots moving away from the core in a precessing nozzle. The outer structure
( $\sim 1.5$ mas) can also be fitted into this picture if the velocity of the ballistic components decrease with distance.

We thank the referee for suggestions that greatly helped to improve the presentation of the paper. We would also like to thank A. V. R. Silva for reading the paper. This research has made use of the United States Naval Observatory Radio Reference Frame Image Database.
Abraham, Z. 2000, A\&A, 355, 915

Collin, S., Mouchet, M., Dumont, A.-M., Coupé, S., Porquet, D., \& Rokaki, E. 2002, A\&A, 388, 771

Denn, G. R., Mutel, R. L., \& Marscher, A. P. 2000, ApJS, 129, 61

Fey, A. F., \& Charlot, P. 1997, ApJS, 111, 95 2000, ApJS, 128, 17

Fey, A. F., Clegg, A. W., \& Fomalont, E. B. 1996, ApJS, 105, 299

Gabuzda, D. C., \& Gómez, J. L. 2001, MNRAS, 320, L49

Hardee, P. E. 1987, ApJ, 318, 78

Homan, D. C., Ojha, R., Wardle, J. F. C, Roberts, D. H., Aller, M. F., Aller, H. D., \& Hughes, P. A. 2001, ApJ, 549, 840

Jorstad, S. G., Marscher, A. P., Mattox, J. R., Wehrle, A. E., Bloom, S. D., \& Yurchenko, A. V. 2001, ApJS, 134, 181

Katz, J. I. 1997, ApJ, 478, 527

Kollgaard, R. I., Wardle, J. F. C., Roberts, D. H., \& Gabuzda, D .C. 1992, AJ, 104,1687

Liu, F. K., \& Wu, X. B. 2002, A\&A, 388, L48

Lu, J. F. 1990, A\&A, 229, 424

Napier, P. J., Bagri, D. S., Clark, B. G., Rogers, A. E. E., Romney, J. D., Thompson, A. R., \& Walker, R. C. 1994, Proc. IEEE, 82, 658

\section{REFERENCES}

Perlman, E. S., \& Stocke, J. T. 1994, AJ, 108, 56

Pursimo, T., et al. 2000, A\&AS, 146, 141

Roberts, D. H., Gabuzda, D. C., \& Wardle, J. F. C. 1987, ApJ, 323, 536

Rogers, A. E. E., et al., 1983, Science, 219, 51

Sarazin, C. L., Begelman, M. C., \& Hatchett, S. P. 1980, ApJ, 238, L129

Shepherd, M. C. 1997, in ASP Conf. Ser. 125, Astronomical Data Analysis Software and Systems VI, ed. G. Hunt \& H. E Payne (San Francisco: ASP), 77

Sillanpää, A., Haarala, S., Valtonen, M. J., Sundelius, B., \& Byrd, G. G. 1988, ApJ, 325, 628

Tateyama, C. E., Kingham, K. A., Kaufmann, P., \& de Lucena, A. M. P. 2002, ApJ, 573, 496

Tateyama, C. E., Kingham, K. A., Kaufmann, P., Piner, B. G., Botti, L. C. L., \& de Lucena, A. M. P. 1999, ApJ, 520, 627

Tateyama, C. E., Kingham, K. A., Kaufmann, P., Piner, B. G., de Lucena, A. M. P., \& Botti, L. C. L. 1998, ApJ, 500, 810

Valtaoja, E., Teräsranta, H., Tornikoski, M., Sillanpää, A., Aller, M. F., Aller, H. D., \& Hughes, P. A. 2000, ApJ, 531, 744

Vicente, L., Charlot, P., \& Sol, H. 1996, A\&A, 312, 727 